\title{
Sensibilidad y especificidad de los análisis de psicotropos para el diagnóstico de los trastornos mentales debidos a su consumo
}

\author{
Bertolín Guillén, J.M. \\ Unidad de psiquiatría, Hospital General Universitario. Diputación Provincial de Valencia.
}

Enviar correspondencia a:

Dr.José Manuel Bertolín, Psiquiatra. Unidad de Psiquiatría, Hospital General Universitario, Avda. Tres Cruces s/n. 46014 Valencia.

\begin{abstract}
Resumen
Objetivos: a) Evaluar los análisis de alcohol, opioides, cocaína, anfetaminas y cannabinoides como pruebas predictoras de los diagnósticos correspondientes de trastornos mentales debidos a psicotropos; y b) Comparar el valor de esos análisis con el autoinforme, respecto del verdadero consumo reciente, para establecer los diagnósticos de trastornos mentales debidos a psicotropos. Pacientes y método: estudio transversal sobre 240 sujetos distintos ingresados consecutivamente en la Unidad de Psiquiatría del hospital en el periodo de mayo a diciembre de 1996. El alcohol se determinó en sangre mediante la tecnología de atenuación de la energía de radiación (REA $\left.{ }^{T M}\right)$, y el resto se determinó en orina mediante el sistema denominado ADX'M . Los análisis se hicieron en las primeras 24 horas del ingreso. Resultados y discusión: Se han hallado 89 trastornos mentales debidos a psicotropos. Todos los análisis de psicotropos se han revelado adecuados para identificar los individuos que no sufren un trastorno mental debido a psicotropos, pero sólo el de opioides identifica bien los trastornos mentales correspondientes. Los diagnósticos de trastornos mentales debidos a psicotropos correlacionan aceptablemente, para los opioides y cocaína, con el consumo positivo autoinformado respecto a la semana previa al ingreso (Kappa $=0,73$ y 0,62, respectivamente), y para los opioides correlacionan también con los análisis respectivos (Kappa = 0,65). Conclusiones: los análisis sistemáticos de psicotropos no proporcionan información clínicamente útil para establecer diagnósticos de trastornos mentales debidos a psicotropos, aunque apoyan complementariamente a la entrevista informativa en el caso de los opioides.
\end{abstract}

Palabras clave: Alcohol, cannabis, opioides, anfetaminas, cocaína, estudio transversal, sensibilidad, especificidad.

\section{INTRODUCCIÓN*}

n los ingresos psiquiátricos de algún hospital se han hallado alcohol y otros psicotropos mediante análisis de orina en el $17,45 \%$ de los mismos (1).

\begin{abstract}
Aims: a) To evaluate the analysis for alcohol, opiates, cocaine, amphetamines and cannabinoids as predictive tests for the corresponding current diagnosis of mental disorders due to psychotropic drugs (MDPD). b) To compare the value of those analysis with recent consumption self report respecting the true recent consumption in order to establish the diagnoses of MDPD. Patients and method: Cross study on 240 different subjects consecutively admitted to the Unit of Psychiatry of the hospital within the period from May to December 1996. Alcohol was determined in blood by means of the radiation energy attenuation technology (REA $\left.{ }^{\mathrm{TM}}\right)$. The others were determined in urine by means of the system called ADX ${ }^{\mathrm{TM}}$. The analysis were performed within the first 24 hours after admission. Results and discussion: 89 MPDP were found. All the analysis for psychotropic drugs are appropriated to identify those individuals who are not suffering from a MDPD, but only the analysis for opiates identifies properly the corresponding MDPD. The diagnoses of MDPD acceptably correlate, for opiates and cocaine, with the self-reported positive consumption with respect to the week before admission (Kappa $=0.73$ and 0.65 respectively). For opiates, the diagnoses also correlate with the respective analysis (Kappa=0.65). Conclusions: The systematic analysis of psychotropic drugs do not give clinically useful information in order to establish diagnoses of MDPD, though they are a complementary help to the informative interview in the case of opiates.
\end{abstract}

Key word: Alcohol, cannabis, opiates, amphetamines, cocaine, cross study, sensitiveness, specificity.

\footnotetext{
* El sustantivo "psicotropos" se usará en el presente trabajo como sinónimo de la expresión "sustancias psicotropas" utilizada por la CIE10 (Organización Mundial de la Salud, 1992), sinónimo también de la expresión más específica "sustancias asociadas a trastornos" utilizada por el DSM-IV (Asociación Americana de Psiquiatría, 1994) que a su vez sustituía a la más imprecisa "sustancias psicoactivas" empleada antes por el DSM-III-R (1987). Por otra parte, la palabra "análisis" será sinónimo de "análisis de laboratorio", usándose indistintintamente ambas expresiones para indicar el análisis clínico de los distintos psicotropos, salvo cuando se especifique en el texto otro significado (por ejemplo "análisis descriptivo" aplicado a la muestra).
} 
pacientes psiquiátricos generales del 32,43\% (2). Desconocíamos entonces, sin embargo, la sensibilidad y especificidad de los análisis de laboratorio como elementos predictores de los trastornos mentales debidos al consumo de sustancias psicotropas en nuestro medio. También desconocíamos el valor comparado de tales análisis con otras fuentes alternativas de información toxicológica, principalmente el consumo reciente autorreconocido. En este trabajo se intentará responder a estas cuestiones, tras haber avanzado en otro lugar algunos resultados preliminares al respecto (3).

Sobre la base de observaciones propias anteriores (2) sería esperable obtener en la población de ingresos psiquiátricos de la muestra una sensibilidad entre los análisis en orina para el cannabis, cocaína, anfetaminas y opioides y los trastornos mentales debidos al consumo correspondiente de esas sustancias, mayor que la sensibilidad esperable para la alcoholemia. Otros autores, en cambio, han informado una sensibilidad mayor para el alcohol que para el resto de los psicotropos estudiados (4).

Cabe preguntarse si tiene realmente interés clínico conocer la sensibilidad, especificidad y valor predictivo de los análisis de las sustancias asociadas a trastornos mentales que estudiamos en este trabajo, con el fin de diagnosticar los eventuales trastornos mentales que pudieran estar presentes debidos a las mismas. Para el autor la respuesta es afirmativa, pues es preciso mejorar el reconocimiento de los trastornos mentales debidos a psicotropos entre los pacientes psiquiátricos (10). Tal necesidad está apoyada al menos en cuatro evidencias: 1) La elevada prevalencia de comorbilidad de otros trastornos mentales con los debidos específicamente a psicotropos en los hospitales generales (5); 2) La tendencia a infradiagnosticar tanto los patrones de consumo de psicotropos menos problemáticos (6), como los propios trastornos mentales debidos a psicotropos (7), en especial cuando el recurso asistencial no es propiamente una unidad de desintoxicación o no dispone de ella, como es el caso; 3) La reducida fiabilidad de las autorrevelaciones acerca del consumo cualitativo de sustancias psicotropas por parte de muchos pacientes psiquiátricos $(1,8,9)$; y 4) La conveniencia de sospechar algún trastorno mental por psicotropos cuando el paciente que demanda asistencia por otro trastorno mental evoluciona insatisfactoriamente de este último. Con el presente trabajo se pretende establecer pues qué cantidad de información clínicamente útil para el diagnóstico de los trastornos mentales debidos a psicotropos añade un resultado positivo o negativo de los análisis para cada sustancia.

\section{PACIENTES Y MÉTODO}

La investigación se ha llevado a cabo en la Unidad de Psiquiatría del Hospital General Universitario, de
Valencia. La Unidad dispone de 20 camas y atiende las hospitalizaciones de psiquiatría general de un área territorial de 336.823 habitantes. No se admiten en la Unidad los pacientes que pretenden una desintoxicación de psicotropos, para la que hay otras unidades específicas de desintoxicación en otros centros hospitalarios. La condición de ser solamente toxicómano excluía pues al paciente de ingresar en nuestra Unidad, salvo que hubiera otro trastorno mental comórbido por el que fuera conveniente el ingreso. La población del estudio está formada por los pacientes que han ingresado consecutivamente en la Unidad durante el periodo de mayo a diciembre de 1996, N= 240. Cada paciente sólo está representado en una ocasión, la que corresponde a su primer o único ingreso en ese periodo. A todos ellos se les ha practicado en las primeras 24 horas del ingreso un análisis de orina para detectar cannabinoides, cocaína, anfetaminas y opioides, y de sangre para determinar la alcoholemia.

Los análisis en orina y sangre se realizaron en el área de Farmacocinética Clínica del Servicio de Farmacia del hospital. En las muestras de orina se utilizó el sistema denominado $A D X^{\mathrm{TM}}$ para la detección de psicotropos, basado en una metodología de inmunoensayo. Los resultados se expresaron en términos cualitativos como positivo o negativo, según fuesen superiores o inferiores a una concentración umbral establecida (11). El alcohol se determinó en las muestras de sangre mediante la tecnología de atenuación de la energía de radiación (REA $A^{\mathrm{TM}}$ ) y los principios fundamentales de la ley de Beer. Los tiempos de detección de los distintos psicotropos varían desde 24 horas para el alcohol hasta varias semanas para los cannabinoides.

Para los diagnósticos de los trastornos mentales debidos a psicotropos se ha seguido la clasificación de la CIE-10 en su versión para la investigación (12). Las cinco categorías de trastornos mentales debidos a psicotropos que estudiamos se corresponden con los códigos diagnósticos de la CIE-10: F10, F11, F12, F14 y F15, integrados todos ellos en el grupo categórico de "trastornos mentales y del comportamiento debidos al consumo de sustancias psicotropas." Cada categoría diagnóstica incluye trastornos diversos, desde la intoxicación no complicada y el consumo perjudicial hasta cuadros psicóticos y de demencia manifiestos, pero todos ellos secundarios al consumo de alguna sustancia psicotropa. Para mejorar la fiabilidad diagnóstica se ha usado además un cuestionario de elaboración propia, derivado de los cuestionarios de las secciones 11 y 12 del sistema "SCAN" para la evaluación clínica en neuropsiquiatría (13). Todos los diagnósticos están referidos al episodio actual, que abarca usualmente desde el mes anterior al ingreso. Los diagnósticos lo son al alta hospitalaria. El criterio de "verdad" respecto del consumo de sustancias psico- 
tropas en nuestro cuestionario viene dado por un ítem que expresa el juicio de un psiquiatra experimentado resultante de unir todas las fuentes disponibles de información al respecto (análisis, autoinformes e informes de terceros, principalmente). Este criterio de verdad se tipifica como "consumo durante la última semana" para indicar un consumo cierto. El estudio estadístico de los datos se ha realizado con el programa informático SPSS ${ }^{\text {TM }}$. El resultado dicotómico de los análisis en orina y sangre será la variable predictora, y la presencia o no de trastorno mental debido a psicotropos la variable de desenlace.

\section{Descripción de los indicadores usados}

La sensibilidad y especificidad de cada análisis de laboratorio la hemos calculado de acuerdo con las siguientes igualdades: Sensibilidad= PV:(PV+FN); Especificidad= NV:(NV+FP). El valor predictivo de un análisis con resultado positivo (VP+) y negativo (VP-) se calcula como sigue: VP+= PPV:(PPV+PFP); VP-= PNV:(PNV+PFN). Donde PV es los resultados positivos verdaderos (análisis positivo en un sujeto con un trastorno mental debido al mismo psicotropo), FN los falsos negativos, NV los negativos verdaderos, FP los falsos positivos, PPV la probabilidad de un resultado positivo verdadero, PFP la probabilidad de un resultado falso positivo, PNV la probabilidad de un resultado negativo verdadero y PFN la probabilidad de un resultado falso negativo.

El estadígrafo prevalencia o probabilidad previa se define como el número de sujetos con trastorno mental debido psicotropos en relación al número de sujetos de la muestra. La razón de probabilidad o verosimilitud es la probabilidad de que un sujeto con un trastorno mental debido a algún psicotropo presente un resultado positivo en el análisis correspondiente, dividido por la probabilidad de que un sujeto sin dicho trastorno mental muestre el mismo resultado. La odds preprueba para un trastorno mental debido a psicotropos se determina como el cociente entre la proporción de sujetos con el trastorno y la proporción de sujetos sin dicho trastorno. Para determinar la odds posprueba de un trastorno dado se multiplican la odds preanálisis de orina o sangre por la razón de probabilidad del resultado de ese mismo análisis.

Por último, para estimar la fiabilidad de los datos observacionales entre las distintas fuentes de información además de los análisis, se usará el estadígrafo Kappa de Cohen, que representa la razón entre el acuerdo observado y no azaroso dividido por el posible acuerdo total no producido por azar. El valor mínimo aceptable es 0,60 y se considerará excelente por encima de 0,75. Para la prueba ji-cuadrado de significación estadística la hipótesis nula será que los resultados se deban sólo a la variación muestral.

\section{RESULTADOS}

\section{Análisis descriptivo de la muestra}

Las características de la población por sus variables sociodemográficas principales se describen en la tabla I. Los 240 ingresos han generado al alta 366 diagnósticos de trastornos mentales, 245 no debidos a psicotropos y 121 debidos a psicotropos (66,94\% y $33,06 \%$, respectivamente, excluidos los debidos al tabaco). Los trastornos mentales debidos al alcohol, opioides, cannabinoides, anfetaminas y cocaína, que son cuantos analizamos en este trabajo, suman 89 y representan el $24,32 \%$ de todos los diagnósticos de trastornos mentales.

\section{TABLA I \\ CARACTERISTICAS SOCIODEMOGRAFICAS DE LOS PACIENTES DE LA MUESTRA ( $\mathrm{N}=\mathbf{2 4 0}$ )}

\begin{tabular}{lrr}
\hline & & VALOR \\
\hline EDAD & & \\
& MINIMA & 14 \\
MAXIMA & 74 \\
MEDIA & 36,64 \\
MEDIANA & 34 \\
MODA & 29 \\
& DESV. EST. & 13,36
\end{tabular}

SEXO

$\begin{array}{lrr}\text { VARONES } & 149 & 62,08 \\ \text { MUJERES } & 91 & 37,92\end{array}$

ESTADO CIVIL

$\begin{array}{lll}\text { SOLTEROS } & 152 & 63,33\end{array}$

CASADOS

VIUDOS

SEPARADOS

8,75

MODO CONVIVENCIA

$\begin{array}{rrr}\text { SOLO } & 26 & 10,83 \\ \text { FAMILIA } & 183 & 76,25 \\ \text { GRUPO NO FAMILIAR } & 12 & 5,00 \\ \text { TRANSEUNTE } & 8 & 3,33 \\ \text { OTROS } & 11 & 4,58\end{array}$

SITUACION LABORAL

EMPLEADO

34

14,17

DESEMPLEADO

PENSIONISTA

ESTUDIANTE

HOGAR

OTROS

31,67

39,58

2,50

11,67

0,42

RESIDENCIA

VALENCIA $\quad 100 \quad 41,67$

RESTO DEL AREA $\quad 140 \quad 58,33$ 


\section{TABL A II. VALORACION DE LOS ANALISIS DE PSICOTROPOS COMO PRUEBAS DIAGNOSTICAS}

DETRASTORNOS MENTALES POR PSICOTROPOS

\begin{tabular}{|c|c|c|c|c|c|c|c|c|c|c|c|c|c|}
\hline \multirow{2}{*}{$\begin{array}{l}\text { SUSTANCIAS } \\
\text { PSICOTROPAS }\end{array}$} & \multicolumn{2}{|c|}{$\begin{array}{c}\text { TRAS.MENTAL } \\
\text { F10-19 }\end{array}$} & \multicolumn{4}{|c|}{ ANALISIS } & \multirow[t]{2}{*}{$\begin{array}{r}\text { SENSI- } \\
\text { BILIDAD }\end{array}$} & \multirow[t]{2}{*}{$\begin{array}{l}\text { ESPECl- } \\
\text { FICIDAD }\end{array}$} & \multirow{2}{*}{$\begin{array}{l}\text { VALOR } \\
\text { PREDICT. } \\
\text { POSIT. }\end{array}$} & \multirow{2}{*}{$\begin{array}{c}\text { VALOR } \\
\text { PREDICT. } \\
\text { NEGAT }\end{array}$} & \multirow{2}{*}{$\begin{array}{l}\text { RAZÓN } \\
\text { PROBA- } \\
\text { BILIDAD }\end{array}$} & \multirow{2}{*}{$\begin{array}{l}\text { ODDS } \\
\text { PRE } \\
\text { PRUEBA }\end{array}$} & \multirow{2}{*}{$\begin{array}{c}\text { ODDS } \\
\text { POST } \\
\text { PRUEBA }\end{array}$} \\
\hline & $\mathrm{N}$ & PREV. & PV & $\mathrm{FN}$ & NV & FP & & & & & & & \\
\hline 1. $\mathrm{ALCOHOL}$ & 39 & 0,16 & 18 & 21 & 184 & 17 & 0,46 & 0,92 & 0,51 & 0,90 & 5,46 & 0,19 & 1,06 \\
\hline 2. CANNABIS & 20 & 0,08 & 10 & 10 & 195 & 25 & 0,50 & 0,89 & 0,29 & 0,95 & 4,40 & 0,09 & 0,40 \\
\hline 3. OPIOIDES & 12 & 0,05 & 9 & 3 & 222 & 6 & 0,75 & 0,97 & 0,60 & 0,99 & 28,50 & 0,05 & 1,50 \\
\hline 4. ANFETAMINAS & 9 & 0,04 & 1 & 8 & 225 & 6 & 0,11 & 0,97 & 0,14 & 0,97 & 4,28 & 0,04 & 0,17 \\
\hline 5. COCAINA & 9 & 0,04 & 3 & 6 & 224 & 7 & 0,33 & 0,97 & 0,30 & 0,97 & 11,00 & 0,04 & 0,43 \\
\hline Total: & 89 & 0,37 & & & & & & & & & & & \\
\hline $2+3+4+5$ & 50 & 0,21 & 23 & 27 & 866 & 44 & 0,46 & 0,95 & 0,71 & 0,87 & 9,51 & 0,26 & 2,50 \\
\hline
\end{tabular}

NOTAS = F10-19: códigos de la CIE-10 correspondientes a los Trastornos mentales y del comportamiento debidos al consumo de sustancias psicotropas. PV: positivos verdaderos; FN: falsos negativos; NV: negativos verdaderos; FP: falsos positivos.

Si consideramos todos los posibles trastornos mentales debidos a psicotropos con excepción de los debidos al tabaco, es decir, añadiendo a los anteriores los sedantes, otros estimulantes, alucinógenos y otros psicotropos, el total hallado en la muestra es 121. Así pues, los trastornos mentales debidos a psicotropos de los que nos ocuparemos en este trabajo representan el 73,55\% de esos diagnósticos, según son categorizados en la CIE-10.

\section{Sensibilidad y especificidad}

La proporción de individuos ingresados en la Unidad de Psiquiatría con trastornos mentales debidos al alcohol con alcoholemia positiva al ingreso (sensibilidad) es 0,46, y la proporción de individuos sin tales trastornos que presentan un análisis de alcoholemia negativo (especificidad) es 0,92 (tabla II). Por tanto, la probabilidad de que una persona ingresada con alcoholemia positiva sufra un trastorno mental debido al alcohol (valor predictivo de un análisis con resultado positivo) es 0,51 , y la probabilidad de que una persona con un resultado negativo no sufra en realidad un trastorno mental debido al alcohol (valor predictivo de un análisis con resultado negativo) es 0,90. La probabilidad de que una persona diagnosticada de un trastorno mental debido al alcohol presente alcoholemia positiva (razón de probabilidad) es 5,46. La probabilidad previa al análisis de alcoholemia de sufrir un trastorno mental debido al alcohol es 0,19 y, finalmente, la probabilidad posterior al análisis positivo es 1,06.

Análogo análisis descriptivo es aplicable al resto de psicotropos. Agrupando los resultados obtenidos para el cannabis, opioides, anfetaminas y cocaína se pueden ver tales resultados en la última fila de la tabla II. La sensibilidad para todos ellos es igual que para el alcohol, y muy similar la especificidad. El valor predic- tivo positivo mejora en el conjunto de los cuatro psicotropos agrupados en relación al del alcohol debido sobre todo a la mayor prevalencia de los trastornos mentales debidos a psicotropos para la suma de esos psicotropos (0,21 frente a 0,16 para el alcohol).

El valor predictivo negativo se mantiene muy similar tanto para el conjunto de los psicotropos agrupados como para cada uno de ellos en relación al del alcohol. La razón de probabilidad mejora para el agrupamiento de los cuatro por efecto de la elevada razón correspondiente a los opioides y cocaína. La mejor odds preprueba se da en el alcohol frente a cada uno del resto de psicotropos, pero al agruparlos mejora en el conjunto frente a aquél. Por último, también es mejor la odds posprueba en el conjunto agrupado en relación a la del alcohol.

\section{Coeficientes de fiabilidad}

La correlación entre los análisis positivos y el verdadero consumo durante la última semana es aceptable para los opioides, cannabinoides y cocaína (tabla III), y es igualmente aceptable entre los análisis y el consumo autorreconocido para los mismos psicotropos. Los diagnósticos de trastornos mentales debidos a opioides y cocaína correlacionan aceptablemente con el consumo autorreconocido en la semana previa al ingreso, y para los opioides correlacionan además con el consumo verdadero durante la última semana y con los análisis. EI índice de Kappa es aceptable entre el consumo autorreconocido y el consumo verdadero durante la última semana para el alcohol y anfetaminas, y es óptimo para los opioides, cannabinoides y cocaína. Para todos los valores Kappa obtenidos es despreciable la probabilidad que tiene la hipótesis nula de ser correcta, si realmente dicha hipótesis fuese cierta. 
TABLA III. COEFICIENTES DE FIABILIDAD KAPPA ENTRE DISTINTOS JUICIOS

\begin{tabular}{|c|c|c|c|c|}
\hline & & $\begin{array}{l}\text { CONSUMO } \\
\text { ULTIMA } \\
\text { SEMANA }\end{array}$ & $\begin{array}{l}\text { ANALISIS } \\
\text { POSITIVO }\end{array}$ & $\begin{array}{l}\text { TRASTORNO } \\
\text { MENTAL POR } \\
\text { PSICOTROPOS }\end{array}$ \\
\hline ALCOHOL & $\begin{array}{l}\text { CONSUMO AUTORRECONOCIDO } \\
\text { CONSUMO ULTIMA SEMANA } \\
\text { ANALISIS POSITIVO }\end{array}$ & $\begin{array}{c}0,69811 \\
- \\
-\end{array}$ & $\begin{array}{c}0,39004 \\
0,38228 \\
-\end{array}$ & $\begin{array}{l}0,40389 \\
0,46867 \\
0,39321\end{array}$ \\
\hline OPIOIDES & $\begin{array}{l}\text { CONSUMO AUTORRECONOCIDO } \\
\text { CONSUMO ULTIMA SEMANA } \\
\text { ANALISIS POSITIVO }\end{array}$ & $\begin{array}{c}0,81028 \\
- \\
-\end{array}$ & $\begin{array}{c}0,78667 \\
0,74704 \\
-\end{array}$ & $\begin{array}{l}0,72549 \\
0,69072 \\
0,64706\end{array}$ \\
\hline CANNABINOIDES & $\begin{array}{l}\text { CONSUMO AUTORRECONOCIDO } \\
\text { CONSUMO ULTIMA SEMANA } \\
\text { ANALISIS POSITIVO }\end{array}$ & $\begin{array}{c}0,80226 \\
- \\
-\end{array}$ & $\begin{array}{c}0,66083 \\
0,72464 \\
-\end{array}$ & $\begin{array}{l}0,45806 \\
0,53333 \\
0,28814\end{array}$ \\
\hline COCAINA & $\begin{array}{l}\text { CONSUMO AUTORRECONOCIDO } \\
\text { CONSUMO ULTIMA SEMANA } \\
\text { ANALISIS POSITIVO }\end{array}$ & $\begin{array}{c}0,96076 \\
- \\
-\end{array}$ & $\begin{array}{c}0,68061 \\
0,64964 \\
-\end{array}$ & $\begin{array}{l}0,6195 \\
0,58998 \\
0,28767\end{array}$ \\
\hline ANFETAMINAS & $\begin{array}{l}\text { CONSUMO AUTORRECONOCIDO } \\
\text { CONSUMO ULTIMA SEMANA } \\
\text { ANALISIS POSITIVO }\end{array}$ & $\begin{array}{c}0,66038 \\
- \\
-\end{array}$ & $\begin{array}{c}0,34985 \\
0,48754 \\
-\end{array}$ & $\begin{array}{l}0,13386 \\
\mathrm{Ns} \\
0,09532\end{array}$ \\
\hline
\end{tabular}

$p=0,00000$ para todos los valores. Ns= no significativo. En cursiva los valores del coeficiente Kappa por encima de 0,60 .

\section{DISCUSIÓN}

Como referencia recordemos que se ha estimado una sensibilidad y especificidad del cuestionario CAGE para detección de alcohólicos en atención primaria del $69 \%$ y $80 \%$ respectivamente (14), mientras que en el presente trabajo la sensibilidad y especifidad de la alcoholemia positiva para determinar un trastorno mental debido al alcohol es del $46 \%$ y $92 \%$ respectivamente. Esperábamos hallar menor sensibilidad relativa para los análisis de alcohol que para el resto de psicotropos, pero el resultado es similar cuando agrupamos dicho resto. Por separado, la mayor sensibilidad es para los opioides (75\%), seguido de los cannabinoides (50\%). Los resultados de las anfetaminas y cocaína, aparentemente contradictorios con la hipótesis de partida que se formulaba en la introducción, se explican por la importancia del número de falsos negativos en ambos. Un resultado positivo a opioides tiene, por tanto, el mayor valor predictivo de todos para establecer un diagnóstico de trastorno mental debido a su consumo $(60 \%)$, predicción que es superior a la del alcohol (51\%). El valor predictivo de los análisis de las otras sustancias es bajo. Dada la mayor diferencia entre la probabilidad previa y posterior a los análisis de opioides de sufrir un trastorno mental debido a los mismos, dichos análisis sí aportan en principio una información válida para considerar el diagnóstico correspondiente.

Hipotetizábamos también mayor especificidad para el alcohol, obteniendo en cambio resultados similares pero ligeramente inferiores en relación con el resto. La explicación debe buscarse en la elevada frecuencia relativa de falsos positivos para casi todos los psicotropos analizados, incluyendo el alcohol. La frecuencia de falsos positivos es máxima en el caso de los cannabinoides $(25 \%$ de falsos positivos frente a $10 \%$ de positivos verdaderos), por lo que la especificidad es mínima en éstos. Pero la elevada frecuencia de falsos positivos para los trastornos mentales debidos a psicotropos no debe hacer olvidar que los consumos detectados de las sustancias muy probablemente influyen sobre los pacientes ingresados empeorando o distorsionando los síntomas de presentación de los demás trastornos mentales, que son precisamente por los que son ingresados $(2,5)$. En este sentido se ha informado que las urgencias relacionadas con el alcohol pueden llegar a ser la cuarta causa en importancia de todas las urgencias psiquiátricas de un hospital general (15). Por tanto, aún cuando el resultado positivo del análisis no se corresponda con un diagnóstico de trastorno mental debido a esa sustancia la 
información será al menos clínicamente útil para el manejo a corto plazo del paciente psiquiátrico que ingresa en la Unidad.

Cuando comparamos entre sí las distintas fuentes alternativas de información toxicológica, los análisis sólo han correlacionado significativa y discretamente ( $p=0,00000$; índice de Kappa=0,64706) con los diagnósticos de trastorno mental por opioides, mientras que el consumo autorreconocido correlaciona con los trastornos mentales debidos opioides y cocaína. Las dos fuentes de información parecen, en consecuencia, poco determinantes a la hora de establecer un diagnóstico de trastorno mental debido al alcohol, cannabinoides y anfetaminas. La mayor concordancia en todos los casos se da entre la autoinformación de un consumo positivo durante la última semana y el verdadero consumo habido en ese tiempo. Los pacientes tienden pues a informar bastante verazmente del consumo reciente de las cinco sustancias analizadas, lo que parece contrastar con lo informado por otros autores $(1,8)$; Pero en cualquier caso esa autorrevelación no correlaciona con que se dé un verdadero trastorno mental debido al alcohol, cannabinoides o anfetaminas. Por otra parte nuestros resultados se pueden explicar por la carga probatoria que para establecer el consumo verdadero tiene el autorreconocimiento del mismo en el cuestionario que hemos empleado.

Los análisis son pues poco fiables para establecer diagnósticos de trastornos mentales debidos a psicotropos en todos los casos excepto para los opioides; Ahora bien, incluso para éstos su correlación es inferior al autorreconocimiento del sujeto, de modo que no proporcionan información adicional que sea clínicamente útil para un diagnóstico. Por otro lado el autorreconocimiento del consumo correlaciona también mal con los trastornos mentales debidos al alcohol, cannabinoides y anfetaminas; Es aceptable para la cocaína, superando a los análisis; Es buena, por último, como se señalaba antes, para los opioides, por encima de la información proporcionada por los análisis.

Aunque la población del estudio es reducida y la potencia del trabajo es por tanto también reducida, representa la totalidad de la población real para el periodo abarcado, lo que le confiere validez. A la luz de los resultados puede pues concluirse que todos los análisis de psicotropos son buenos para identificar los individuos que no sufren un trastorno mental debido a psicotropos, pero sólo el de opioides identifica los correspondientes trastornos mentales presentes. En efecto, los análisis de opioides tienen la mayor sensibilidad y comparten la mayor especificidad entre los distintos análisis, su valor predictivo negativo es también el mayor, así como lo es la razón de probabilidad y la odds posprueba. Pero el índice de Kappa de los análisis de opioides con los trastornos mentales debi- dos a su consumo, aunque es suficiente, es inferior al mero autorreconocimiento del consumo del sujeto. También el autorreconocimiento de un consumo de cocaína durante la última semana parece más concordante con un trastorno mental debido a dicho consumo que el resultado positivo de los análisis. Así pues el presente trabajo fracasa en poder evidenciar que los análisis superen al autorreconocimiento del consumo para establecer un diagnóstico correcto de cualquier trastorno mental debido a los cinco psicotropos estudiados. En consecuencia, los análisis sistemáticos al ingreso de las cinco sustancias psicotropas no proporcionan una información adicional clínicamente útil cara a establecer diagnósticos de trastornos mentales debidos a psicotropos, aunque apoyan complementariamente a una buena entrevista informativa en el caso de los debidos a opioides.

\section{AGRADECIMIENTO}

A Amparo Muñoz, del Centro de Investigación del hospital, por la lectura crítica del borrador. Y a cuantas personas han hecho posible esta investigación con su trabajo o recogida de información.

\section{BIBLIOGRAFÍA}

(1) Galletly CA, Field CD, Prior M. Urine drug screening of patients admitted to a state psychatric hospital. Hosp Com Psychiatry 1993; 44: 587-589.

(2) Bertolín Guillén JM, Pretel Piqueras J, Sánchez Hernández A, Acebal Gómez I. Determinación en sangre y orina del consumo reciente no terapéutico de sustancias psicotropas en los pacientes que ingresan en la Unidad de Psiquiatría de un hospital general de Valencia. Rev Esp Salud Publica 1996; 70: 319-329.

(3) Bertolín Guillén JM, Sánchez Hernández A, Pretel Piqueras J, Fdez.-Palacios P, Santos Leal E. Sensibilidad y especificidad de los análisis de psicotropos en orina y sangre en relación con los trastornos mentales por psicotropos, en pacientes de una unidad hospitalaria de psiquiatría. II Congreso Nacional de Psiquiatría, Valencia, 1997. Psiquiatr Biol 1997; 4 (Supl 2): 41.

(4) Albanese MJ, Bartel RL, Bruno RF, Morgenbesser MW, Schatzberg AF. Comparison of mesures used to determine substance abuse in an inpatient psychiatric population. Am J Psychiatry 1994; 151: 1077-1078.

(5) Bertolín Guillén JM, Torres Hernández MA, Pretel Piqueras J, Sánchez Hernández A, Guevara Serrano J. Prevalencia y comorbilidad de trastornos mentales debidos al consumo de psicotropos en una unidad de ingresos psiquiátricos de hospital general. An Psiquiatr 1998; 14: 91-99. 
(6) Pérez K, Domingo-Salvany A, Garcés JM, Hartnoll RL. Información sobre el consumo de opioides y cocaína en la anamnesis de urgencias: validez y fiabilidad. Med Clin (Barc) 1996; 107: 702-705.

(7) Martínez Crespo JM, Antolín E, Comas C, Coll O, Marqués JM, Gual $A$, et al. The prevalence of cocaine abuse during pregnancy in Barcelona. Eur J Obstet Gynecol Reprod Biol 1994; 56: 165-167.

(8) Hoffmann NG, Ninonuevo FG. Concurrent validation of substance abusers self-reports against collateral information: percentage agreement vs. Kappa vs. Yule's Y. Alcohol Clin Exp Res 1994; 18: 231-237.

(9) McPhillips MA, Kelly FJ, Barnes TR, Duke PJ, Gene Cos N, Clark K. Detecting comorbid substance misure among people with schizophrenia in the community: a study comparing the results of questionnaires with analysis of hair and urine. Schizophr Res 1997; 25: 141-148.

(10) Johnson S. Dual diagnosis of severe mental illness and substance misure: a case for specialist services? Br J
Psychiatry 1997; 171: 205-208.

(11) Rawls WN. Urine drug analysis and treatment of substance abuse. Drug Monit Forum 1986; 4: 1-5.

(12) Organización Mundial de la Salud. CIE 10. Décima revisión de la clasificación internacional de las enfermedades. Trastornos mentales y del comportamiento. Criterios diagnósticos de investigación. Madrid: Meditor, 1994.

(13) Organización Mundial de la Salud. SCAN, versión española. Cuestionarios para la evaluación clínica en neuropsiquiatría. Catego 5. Madrid: Meditor, 1993.

(14) Escobar F, Espí F, Canteras M. Diagnostic test for alcoholism in primary health care: compared efficacy of diferent instruments. Drug Alcohol Depend 1995; 40: 151-158.

(15) Simón Lorda D, Gómez-Reino Rodríguez I, Mazaira Castro J, González Vázquez Al, Docasar Bertolo L, Albarrán Barrado MA, et al. Alcoholismo y urgencias psiquiátricas en el hospital general. Adicciones 1996; 8: 19-31. 
